Umstrittene Klimabilanzen von Biokraftstoffen

\title{
Das Dilemma der Europäischen Kommission
}

\begin{abstract}
Verbindliche Kriterien sollen die Klimafreundlichkeit der in Europa genutzten Biokraftstoffe sicherstellen. Vermehrt wird eine Überarbeitung dieser Kriterien gefordert, insbesondere wegen der Effekte indirekter Landnutzungsänderungen. Die EU-Kommission kann diese Forderungen nicht ignorieren.

Allerdings steckt sie dabei in einem Dilemma. Von Thomas Vogelpohl, Elisa Dunkelberg und Bernd Hirschl
\end{abstract}

legte, verbindliche Zehn-Prozent-Ziel angerechnet werden, müssen sie diese Kriterien erfüllen. Durch die Anerkennung von Zertifizierungssystemen können die Kriterien praktisch umgesetzt werden.

\section{Unzureichende Kriterien?}

Diese Entscheidung wurde jedoch von zahlreichen kritischen Stimmen begleitet. Diese beziehen sich allerdings nicht in erster Linie auf die konkrete Entscheidung oder das System der Zertifizierung, sondern vor allem auf die zugrunde liegenden Kriterien selbst. Denn was nützt ein funktionierendes System zum Nachweis der Einhaltung bestimmter Kriterien, wenn die Kriterien selbst unzureichend sind? Genau dies ist nach Ansicht vieler Beobachter der Fall. Neben der Nichtberücksichtigung von Sozialkriterien wird vor allem kritisiert, dass die Kriterien zwar ab 2013 einen bestimmten Mindestreduktionswert von Kohlenstoffdioxid $\left(\mathrm{CO}_{2}\right)$ umfassen, indirekte Landnutzungsänderungen (iLUC; siehe Kasten) bei der Berechnung dieses Wertes jedoch keine Rolle spielen.

Trotz dieser Einwände wurde letztendlich die schlankere Version der Nachhaltigkeitskriterien ohne iLUC beschlossen. Dies geschah nicht zuletzt in Ermangelung geeigneter, wissenschaftlich fundierter Kriterien und Indikatoren. Stattdessen sollte die Kommission bis Ende 2010 einen Bericht zu diesem Aspekt erstellen, auf dessen Grundlage gegebenenfalls Änderungen vorgenommen werden sollten.

\section{Große Spannbreite wissen- schaftlicher Ergebnisse}

In Vorbereitung des iLUC-Berichts gab die Kommission mehrere Studien in Auftrag. Darüber hinaus beschäftigten sich Forscher weltweit mit der Erforschung von Effekten von iLUC. Die Ergebnisse besitzen jedoch eine große Spannbreite, die eine einheitliche Bewertung erschwert. Ursächlich für diese Spannbreite sind zum einen die verschiedenen Berechnungsmethoden und zum anderen zahlreiche unsichere Annahmen, die in die Berechnungen eingehen. Außerdem wirken sich lokale Gegebenheiten auf den Umfang von iLUC aus, die in stark unterschiedlichem Maße Berücksichtigung finden.

Dennoch fordert mittlerweile eine stetig steigende Zahl an Akteuren die Regulierung von iLUC. Unklar ist, auf welche Art und Weise dies geschehen sollte. Aus diesem Grund sah sich die Kommission Ende 2010 nicht in der Lage, sich auf einen regulativen Umgang mit der iLUCThematik festzulegen. Sie „erkennt jedoch an, dass indirekte Landnutzungsänderungen sich auf die Reduktion der mit Biokraftstoffen verbundenen Treibhausgasemissionen auswirken können “ und dass gegebenenfalls regulative Maßnahmen getroffen werden sollten (Europäische Kommission 2010). Die Entscheidung hierzu wurde damals auf den Juli 2011 verschoben.

\section{Möglichkeiten der politischen Regulierung}

Statt eine Entscheidung zu treffen nennt die Kommission vier Optionen zur Regulierung der Effekte von indirekten Landnutzungsänderungen (ebd.):

- zunächst nicht aktiv zu werden,

- die Erhöhung der Mindestschwellwerte für die $\mathrm{CO}_{2}$-Reduktion,

- die Belegung bestimmter Biokraftstoffe mit zusätzlichen Nachhaltigkeitsanforderungen und

ป die Zuweisung geschätzter $\mathrm{CO}_{2}$-Emissionen zu bestimmten Biokraftstoffen, die den Wirkungen von iLUC entsprechen sollen (iLUC-Faktoren). 
Die Diskussionen zum Thema lassen derweil nicht nach. Befeuert werden sie immer wieder durch neue Studien, die teils zu verheerenden Ergebnissen hinsichtlich der Klimabilanz von Biokraftstoffen kommen. Besondere Bedeutung wird hierbei den Studien zugeschrieben, die von der Kommission selbst in Auftrag gegeben wurden. Auch diesen zufolge würde eine Einbeziehung von iLUC die Klimabilanz einzelner Biokraftstoffe, insbesondere von Biodiesel, deutlich verschlechtern. Vor diesem Hintergrund erscheint die erste der oben genannten Möglichkeiten mittlerweile als unwahrscheinlich. Vielmehr zeichnete sich zunächst vor allem die letztgenannte Option als die favorisierte $\mathrm{ab}$.

Das Problem, das sich für die Europäische Kommission daraus ergibt, ist, dass insbesondere viele Biodieselarten die geforderten $\mathrm{CO}_{2}$-Einsparungswerte bei der Integration eines iLUC-Faktors nicht mehr erreichen würden. Allerdings repräsentiert Biodiesel mit 80 Prozent den mit Abstand größten Teil des europäischen Biokraftstoffmarkts. Kann dieser Biodiesel nicht mehr zur Erreichung des ZehnProzent-Ziels verwendet werden, so wird er kaum nachgefragt werden. Die Kommission steckt demnach in dem Dilemma, dass sie einerseits iLUC nicht ignorieren kann, andererseits aber auch die massiven Investitionen wirtschaftlicher Akteure in den Biodieselbereich schützen will, die sie auch durch ihre bisherige Politik heraufbeschworen hat. Vor diesem Hintergrund hatte sie ihre Entscheidung hierzu ein weiteres Mal, auf Ende 2011, verschoben.

\section{Ein fauler Kompromiss?}

Derzeit zeichnet sich jedoch ein Kompromiss ab, der versucht, beiden Seiten gerecht zu werden, indem er die zweite und die vierte der oben genannten Politikoptionen verbindet. Demnach würden biokraftstoffspezifische iLUC-Faktoren eingeführt werden, aber erst im Jahr 2018. Kurzfristig soll dafür die Erhöhung der $\mathrm{CO}_{2}$-Mindestreduktionswerte vorgezogen werden, um die iLUC-Problematik zu adressieren. Der ab 2013 geltende
Ausgangswert soll demnach von 35 auf etwa 45 bis 50 Prozent steigen, und dann ebenfalls früher als geplant im Jahr 2017 auf 60 Prozent steigen.

In die Berechnung dieser Werte würden iLUC dann jedoch nach wie vor nicht einfließen. Diese Regelung hätte für die Biodieselbranche den Vorteil, dass dadurch kurzfristig alle Biokraftstoffe gleichmäßig benachteiligt werden, während die Einführung von iLUC-Faktoren speziell an bestimmte Biodieselarten höhere Anforderungen stellt. Geschieht Letzteres erst 2018, hat die Branche noch Zeit, sich darauf vorzubereiten. Wäre dies nun die salomonische Lösung, die die Wirtschaftlichkeit der europäischen Biodieselbranche und die Klimafreundlichkeit ihrer Produkte in Einklang bringt? Die Branche selbst hat bereits Akzeptanz zu einem solchen Kompromiss signalisiert. Umweltgruppen kritisieren hingegen, dass der tatsächlichen iLUCProblematik, die bei den verschiedenen Biokraftstoffarten nun einmal unterschiedlich relevant sei, damit gar nicht Rechnung getragen und die Problemlösung stattdessen in die Zukunft verschoben würde.

\section{Aussetzung des Ziels als Option}

Das Dilemma wird für die Kommission nicht einfach aufzulösen sein. Will man die iLUC-Problematik ernst nehmen, so müssen letztlich Regelungen getroffen werden, die sie auch tatsächlich adressieren, auch wenn dadurch Teile der Biokraftstoffbranche in Schwierigkeiten geraten könnten. Eine Option, die seitens der Kommission bislang kategorisch abgelehnt wird, ist die Aussetzung oder Abschwächung des verbindlichen Zehn-Prozent-Ziels für Biokraftstoffe. Dabei ist gerade diese Zielmarke mitverantwortlich für den Druck im Biokraftstoffsektor, der sich verstärkt in Verdrängungseffekten (sowohl bezogen auf die Landnutzung als auch auf die Landnutzer) auswirkt, die wiederum die Klima- und Sozialverträglichkeit der Biokraftstoffe beeinträchtigen. Diesen Druck durch eine Abschwächung oder Aussetzung des
Zehn-Prozent-Ziels zumindest teilweise abzulassen, könnte somit, neben weiteren entspannenden Effekten auf die globalen Agrarmärkte, auch eine Abschwächung der iLUC-Problematik auslösen (Gawel/Ludwig 2011).(1)

\section{Anmerkung}

(1) Prinzipiell entstehen iLUC-Effekte im gesamten Agrarbereich und werden vorrangig durch den Fleischkonsum induziert, aber beispielsweise auch durch die Lebensmittel-, Pharmaoder Papierindustrie. Eine biokraftstoffspezifische Regulierung wird daher keine grundsätzliche Lösung des Problems bringen. Vielmehr erfordert es, stärker über eine generelle Regulierung von Agrarprodukten und Maßnahmen zum Erhalt von Wald- und Schutzgebieten auf globaler Ebene nachzudenken.

\section{Literatur}

Europäische Kommission: Bericht der Kommission über indirekte Landnutzungsänderungen im Zusammenhang mit Biokraftstoffen und flüssigen Biobrennstoffen. KOM(2010) 811 endgültig

Gawel, E. / Ludwig, G.: The iLUC dilemma: How to deal with indirect land use changes when governing energy crops? In: Land Use Policy $28,4 / 2011$, S. $846-856$.

I AUTOREN + KONTAKT

Thomas Vogelpohl ist wissenschaftlicher Mitarbeiter, Elisa Dunkelberg ist wissenschaftliche Mitarbeiterin und Bernd Hirschl ist Leiter des Forschungsfeldes Nachhaltige Energiewirtschaft und Klimaschutz am IÖW.

Institut für ökologische Wirtschaftsforschung (IÖW), Geschäftsstelle Berlin, Potsdamer Str. 105, 10785 Berlin. Tel.: +4930 884594-0, Fax.: +49308825493 E-Mail: thomas.vogelpohl@ioew.de, elisa.dunkelberg@ioew.de, bernd.hirschl@ioew.de, Internet: http://www.ioew.de 


\section{Lizenzhinweis}

Die Beiträge in ÖkologischesWirtschaften werden unter der Creative-Commons-Lizenz "CC 4.0 Attribution Non-Commercial No Derivatives" veröffentlicht. Im Rahmen dieser Lizenz muss der Autor/Urheber stets genannt werden, das Werk darf nicht bearbeitet, abgewandelt oder in anderer Weise verändert und außerdem nicht kommerziell genutzt werden. Die digitale Version des Artikels bleibt für zwei Jahre Abonnent/innen vorbehalten und ist danach im Open Access verfügbar. 